\title{
The Role of Multiple Representations and Representational Fluency in Cryptography Education
}

\author{
Ziyang Tang, Xiang Liu, Yingjie Chen, Baijian Yang \\ Purdue University \\ West Lafayette, Indiana \\ \{tang385,xiang35,victorchen,byang\}@purdue.edu
}

\begin{abstract}
Both the public sector and private sector have strong demand for qualified cybersecurity professionals. It is therefore imperative that higher education institutions can fill the gap by producing more skilled graduates that have deep understanding of cybersecurity. Cryptography is one of those fundamental subjects that is difficult to learn and error-prone in practice. This work aims to understand if multiple representations and representational fluency can help students better learn new cryptography concepts. Experiments were designed to present the same new cryptography concepts using three different representations to the participants: language, graph, and math notations. Assessments with different representations were then conducted after the learning phase to associate the learning gains with the learning behaviors. An eye tracker was adopted in this study to keep track of the learning behaviors of the participants'. This IRB-approved study recruited 43 undergraduate students who are majored in IT. Results showed that most participants are language dominant learners. Graphic and math representations are able to help students to improve their comprehensions on new cryptography concepts.
\end{abstract}

\section{CCS CONCEPTS}

- Security and privacy $\rightarrow$ Cryptography; $\bullet$ Social and professional topics $\rightarrow$ Computing education.

\section{KEYWORDS}

Eye Tracking; Cryptography Education; Representational Fluency

\section{ACM Reference Format:}

Ziyang Tang, Xiang Liu, Yingjie Chen, Baijian Yang. 2019. The Role of Multiple Representations and Representational Fluency in Cryptography Education. In The 20th Annual Conference on Information Technology Education (SIGITE '19), October 3-5, 2019, Tacoma, WA, USA. ACM, New York, NY, USA, 6 pages. https://doi.org/10.1145/3349266.3351412

\section{INTRODUCTION}

With the widespread of cyberattacks and our increased reliance on Internet, cybersecurity becomes more and more important for the federal governments, international organizations, and normal

Permission to make digital or hard copies of all or part of this work for personal or classroom use is granted without fee provided that copies are not made or distributed for profit or commercial advantage and that copies bear this notice and the full citation on the first page. Copyrights for components of this work owned by others than ACM must be honored. Abstracting with credit is permitted. To copy otherwise, or republish, to post on servers or to redistribute to lists, requires prior specific permission and/or a fee. Request permissions from permissions@acm.org.

SIGITE '19, October 3-5, 2019, Tacoma, WA, USA

(C) 2019 Association for Computing Machinery.

ACM ISBN 978-1-4503-6921-3/19/10 . \$ \$15.00

https://doi.org/10.1145/3349266.3351412
Internet users. However, there is a significant shortage of highly skilled cybersecurity professionals who have deep understanding of cybersecurity and can design secure systems, as noted by Evans and Reeder [7]. Dale C Rowe et al.[11] estimated that the workforce shortage in the U.S. public sector alone was about 20,000 - 30,000 per year.

To answer the above challenges, higher education must produce sufficient number of security professionals with advanced technical skills. Simms and Chi [12] suggested that a key component in cybersecurity is cryptography and a qualified security practitioner must has a deep understanding in cryptography. One way to improve students' learning outcomes in cryptography is perhaps to apply the theory of representational fluency, as it has demonstrated its effectiveness in many STEM fields. Beckman et al. [2] studied multiple representations and representational fluency in learning cryptography concepts by measuring brain activities using fMRI scans. Although the study shed positive lights on the representational fluency, it lacks statistical significance due to small sample sizes.

In this study, eye-trackers were adopted to observe learner's learning behavior when multiple representations were given. The benefits of using eye-trackers include low cost, low risk, and ease of recruitment of subjects. In the rest of the paper, we review related work in Section 2, followed by elaboration of our design in Section 3. Results are discussed in Section 4 and the paper concludes in Section 5.

\section{LITERATURE REVIEW}

In [10], it is pointed out that a concept can be represented in different formats, such as mathematical, graph, and verbal representations. Each representation creates a different logical model in mind. Representational fluency is defined in [14]. It refers to the ability to translate one form of the representation to another, and hence the ability to transform one logical model to another logical model.

When studying representational fluency in educational settings, two types of techniques are often used: quantitative classroom diagnostics and technology-assisted cognitive process capturing [5]. Quantitative classroom diagnostics usually involves carefully designed surveys on representational fluency. For example, M.Hill et al. [9] solicited students to take a representational fluency survey (RFS). From the results of the survey, the authors found that representational fluency led to a better understanding of the concepts in Physics. In contrast, technology-assisted approach tracks students' cognitive progress. Functional Magnetic Resonance Imaging (fMRI) is one of technologies that was adopted in the literature. Thomas et al.[13] used fMRI and showed that each specific part of the brain is responsible for a specific mental process. J.Beckman et al.[2] also 
applied fMRI in cryptography education. Their research found out that different representations could activate different areas in the brain. When subjects were asked to translate different representation models, longer and stronger brain activities were detected. The fMRI studies can help researchers to better understand the learning process from the neurocognitive perspective, such as how the brain reacts to different representations and what occurs in the brain when translation of representations is needed. Studies using fMRI scans usually incurs high cost. As a result, many of these studies suffered from small sample size problem and were difficult to reach statistically significant results. Eye-tracker [6] is an effective tool that can measure visual attentions and is typically used in usability study. By recording the gaze movement, an eye-tracking machine can report the sequence and the duration of participants' visual attentions. Although eye-trackers are not designed to measure representational fluency, they can certainly be applied to understand the effects of multiple representations if the experiments are designed carefully.

While the previous studies demonstrated that promoting representational fluency could help high school and undergraduate students to gain better understanding in chemistry, physics and mathematics, it is not clear if representational fluency can be applied to the field of cryptography. In addition, it is hard to measure how much improvements was accounted for to the growth of representational fluency. The scope of this project, therefore, focuses on the implicit connections between the mental models created by multiple representations and the understanding of a concept. Specifically, we aimed to investigate how students respond to a new cryptography concept when multiple representations are given. The study also tries to understand if multiple representations are helpful for students to understand the concepts. To this end, we designed a couple of experiments with two cryptography concepts using multiple representations. An eye tracker was used to record participants' gaze movements during the learning process as well as the assessment process. By analyzing the data collected from the eye tracker and the assessment, we were able to associate time allocation and representation transitions with the assessment results.The role of multiple representations can then be inferred from these experiments.

In short, representational fluency has not been extensively applied in the field of cybersecurity. Data collected were either in the format of survey, or the output of sophisticated and expensive machines, like fMRI. A low cost, high validity and high accuracy study is much needed to better understand if and how representational fluency takes the role in cybersecurity education.

\section{METHODOLOGY}

After getting approval from the IRB, we recruited 43 undergraduate students from an entry level undergraduate course in cryptography. Our study aimed at Cryptography education, and therefore the target students should be those learning in the discipline. We chose two advanced Cryptography concepts:

- Zero Knowledge Proof [8]

- Homogeneous Encryption [1]

Both concepts are not covered in the introduction level cryptography class. Presenting new concepts to the participants allows the researchers to observe their learning behaviors, rather than memory recall from their existing knowledge. In addition, these two concepts are not be included the assessment of the entry level cryptography class. Students chose to participate in this study will gain no more advantages than non-participants. Each concept is illustrated in the forms of language, math and graph. Three Assessment questions are created for each concept. Assessment results are used to measure how well the students understand the concept. Each of the three questions is presented in the forms of language, graph and math, respectively.

In our study, the participants were asked to first complete a survey to describe their learning behaviors. The survey results recorded their perceptions on which representation models they prefer the most in learning cryptography concepts. Based on their learning preference, we divided them into Language Dominated Learners, Math Dominated Learners, Graph Dominated Learners and Multiple Representation Learners. A Language Dominated Leaner implies to a student who mainly learns from the language descriptions. But students reported learning preferences may not be the same as their actual learning behaviors. Therefore, the eye tracking experiments were used to observe their learning behaviors and valid the survey results. One of the challenges is that no current technologies can directly observe the translation of different representations. The assumption we made in this study is that transferring from one representation to another is positively correlated to the translating behaviors. In the case of eye tracking, if the learner's focus area moved from the language portion of the display to the math portion of the display, it is denoted as L2M. Likewise, we use the following notations to denote eye movements for the rest combinations: $L 2 G$, G2L, G2M, M2L, M2G.

\subsection{Survey Design}

The main questions composed in the learning behavior survey are as follows:

- Which one of the following (math, graph, language or none of the above) do you prefer the best when learning a concept?

- What kind of representations (math, graph, language) do you usually read first? Why?

- What kind of representations (math, graph, language) do you usually read last? Why?

Based on their answers, we summarized the learning habits of the participants and classified the participants into four groups.A participant was labeled as a self-claimed language dominated learner $(S L D L)$ if she/he prefers language over other representations. Similarly, we have the groups for self-claimed math dominated learner $(S M D L)$ and self-claimed graph dominated learner (SGDL). And if the students chose the option of "None of the above", they will be considered as a self-claimed multiple representation learner (SMRL).

Since we are interested in the role of representational fluency in learning cryptography concepts, we compared the assessment scores of those self-claimed multiple representation learners' (SMRL) against the assessment scores of others' based on their dominated representations. It turned out that for a specific subject, the way how she/he learned the two concepts were not always consistent: a self claimed the language dominated learner (SLRL) could indeed demonstrate the behavior of SLRL for the first concept but showed 


$\begin{array}{c:c}\begin{array}{c}\text { This part will } \\ \text { represent Language }\end{array} & \begin{array}{c}\text { This part will } \\ \text { represent Math }\end{array} \\ \text { This Part will } & \text { This part will } \\ \text { represent Graphs } & \text { Questions }\end{array}$

Figure 1: Divions of the display

the behavior of $S G R L$ in the second concept. To avoid stacking up insistent results, we treated the results from each concept independently. The total number of samples become 86 for the two concepts combined. Results are analyzed in details in Section 4.1

\subsection{Experiment Settings}

We also conducted eye-tracker experiments to observe the learning behaviors when participants studied the new concepts. In the study, the students were given different representations of the new concept on the same page, as shown in Figure 1. The whole screen is divided into $2 * 2$ grids, with each part presents a specific representation model. Since only three representation models were chosen in the study, the last portion of the screen was left empty during the 'learning' phase and was filled with quiz questions during the assessment phase. For each concept, there are four slides. The first slide is concept only. It shows the language representation, the math representation and the graphic representation of the same concept. Essentially, the first slide is the learning phase. Eye tracker was used to study how long each participant spent on each representation and how often the transitions between these representation occurred. Slide \#2 to \#4 are arranged in a similar matter except that the bottom right corner are reserved for quiz questions, in the form of language, math, and graphics, respectively. To minimize the impact of spatial reading habits, for example, many people may have the habbit of reading from left to right and from top to bottom, the position of each types of representation are randomized, and the order of the questions are also randomized.

Each participant completed the experiments at a different pace. The time spent on each tasks should be normalized before analyses. In this study, we used the percentage of the time allocated to each representation as a way to normalize data. Specifically, there are eight slides in the study. The entire eye movement data is therefore breaking into eight chunks, with one chunk of data matching the eye movement of one slide. The eye-tracker samples 1,000 frames evenly per second. Each frame contains the coordinates of the eye focus point $(w, h)$. The location data can be exported as a csv file. At any given moment, to determine which representation (or question) the participant was reading, we can easily map the coordinates (w,h) to the $2^{*} 2$ grids. We use the notation representation percentage $(R P)$ to refer to the percentage of time a specific representation was consumed by a participant. We then defined a threshold, ranging from $50 \%$ to $70 \%$, to classify the participants into specific representation dominated learner. For example, if a participant spends more than $80 \%$ reading the language representations (which is larger than the threshold), she/he is classified as a language dominated learner. The same philosophy applies to the identification of math dominated learners and graph dominated learners. If all of the $R P$ is below the threshold, the participant is classified as a multiple representation learner. However, in the experiment we found some out-liars. A participant may spend most of the time reading language representations when language based questions are given; used most of the time on the graph when graph based questions were presented. There are also some inconsistency between the two concepts. For example, some participants spent most time on language representation for language based question on $Z K P$. But the same participant may spend more time on other representations for the language based question on HE. In this case, instead of dividing the participants into four groups, we treated them as two types of group, i.e. multiple representation learner $(M R L)$ and dominated learner $(D L)$. The dominated learner group includes the participants who solved all the types of questions with only one dominated representation model or solving the specific type of questions with the same type of representations. To be more clearly, for the former case, the participant is labeled as $M R L$ because she/he employed different representations in solving the same type of questions. And in the latter case, the participant is labeled as $D L$ because she/he mainly focused on the same representation to solve that type of questions. More discussions on this can be found in Section 4.2.

Since we are interested in whether the multiple representation learner group has better learning outcome than the representation dominated learner groups, we compared the scores in each specific type of questions accordingly. In addition, we hypothesized that learners who can transfer one representation to another may have better test scores than those who cannot.

\subsection{Hardware and software settings}

The experiments were conducted at a research lab. Each participant was sitting in front of a Windows Desktop with a Tobii Pro X3-120 eye tracker mounted on the top of the monitor. The gaze movement recordings were exported as csv files using Tobii Studio. Python was used to map the coordinates to each representation grid. $\mathrm{R}$ studio was then used for data analyses.

\section{RESULTS AND DISCUSSIONS}

The results of the experiments are illustrated in this section.

\subsection{Study One: Survey Analysis}

From the survey, 55.3\% of the participants considered themselves language dominated learners. $16.3 \%$ of them believed that they were good at learning math. $7.0 \%$ stated they preferred graph examples. Only $11.4 \%$ participants stated that they prefer to use multiple representations when learning new cryptography concepts. Survey results also showed that $69.8 \%$ of the participants would read the language description first and most of them attribute the language description first behavior to habits, something they got used to from K-12 educations. In a world that the knowledge is primarily recorded using text, language first learning behaviors seems to be the natural results to for most learners to adjust to this prevailing representation of knowledge. $62.8 \%$ participants did not like math 
Table 1: Two sample T-test results: MRL v.s. DL $M R L$ vs $L D L$ on Language question, $M R L$ vs $M D L$ on match question, and $M R L$ vs $G D L$ on graphic question.

\begin{tabular}{|c|c|c|c|c|c|c|}
\hline \multicolumn{3}{|c|}{ Group A: MRL } & \multicolumn{3}{|c|}{ Group B: LDL } & \\
\hline $\mathrm{N}$ & mean & std & $\mathrm{N}$ & mean & std & \\
\hline 20 & .45 & .5104 & 46 & .2173 & .4170 & $\begin{array}{c}\mathrm{t}=-1.7942 \\
\mathrm{p}=.0827\end{array}$ \\
\hline \multicolumn{3}{|c|}{ Group A: MRL } & \multicolumn{3}{|c|}{ Group B: MDL } & \\
\hline $\mathrm{N}$ & mean & std & $\mathrm{N}$ & mean & std & \\
\hline 20 & .10 & .3078 & 14 & .3571 & 4972 & $\begin{array}{c}\mathrm{t}=-1.7182 \\
\mathrm{p}=.1013\end{array}$ \\
\hline \multicolumn{3}{|c|}{ Group A: MRL } & \multicolumn{3}{|c|}{ Group B: GDL } & \\
\hline $\mathrm{N}$ & mean & std & $\mathrm{N}$ & mean & std & \\
\hline 20 & .50 & .5129 & 6 & .6667 & .5164 & $\begin{array}{c}\mathrm{t}=.6944 \\
\mathrm{p}=.5066\end{array}$ \\
\hline
\end{tabular}

notations and would prefer to read it as the last resort. They also explained that they didn't enjoy the math notations because these symbols are abstract and difficult to understand.

From these results, we divided the participants into four groups: $S L D L, S M D L, S G D L$ and $S M R L$, as defined in Section 3.1. A twosample T-test was run between the self-claimed multiple representation learners and other three self-claimed dominated learner groups. We compared the scores of the specific type of questions (e.g. the scores of language questions between self-claimed multiple representation learners and self-claimed language dominated learner). The hypothesis we had was that a learner prefers multiple representations can achieve higher test scores than the learners with dominated representations. The results are shown in Table 1.

The results didn't show any statistical significant difference between the self-claimed multiple representation groups and other self-claimed dominated learning groups. We then explored whether self-claimed representation dominated learners have a better understanding on their dominated representations than other groups. And the results are shown in Table 2. The only comparison showed statistical significance was between the self-claimed math dominated learners and the self-claimed graph dominated learners. But in that test, only six samples were in the group of self-claimed graph dominated learners, and none of them answer the math questions correctly. This results may suggest that self-claimed graph dominated learners had difficulties to comprehend abstract math representations. But due to the small sample size, the validity of the results need further investigation.

\subsection{Study Two}

With a hope to reduce the bias from the self-claimed survey, we conducted eye-tracking experiments to capture the eye movement on the screen. As stated in Section 3.2, we divided the participants into multiple representation learner (MRL) group and dominated learner $(D L)$ group. The details are illustrated in Algorithm 1.

In this experiment, the threshold was set to $\beta=50 \%$. This creates multiple representation group of 70 samples, while the group has
Table 2: Two sample T-test results on different representation dominated groups. (The type of of the questions evaluated are the same as the dominated representation of group A)

\begin{tabular}{|c|c|c|c|c|c|c|}
\hline \multicolumn{3}{|c|}{ Group A: LDL } & \multicolumn{3}{|c|}{ Group B: MDL } & \multirow[b]{3}{*}{$\begin{array}{c}\mathrm{t}=-0.9543 \\
\mathrm{p}=.3519\end{array}$} \\
\hline $\mathrm{N}$ & mean & std & $\mathrm{N}$ & mean & std & \\
\hline 46 & .2173 & .4170 & 14 & .3571 & .4972 & \\
\hline \multicolumn{3}{|c|}{ Group A: LDL } & \multicolumn{3}{|c|}{ Group B: GDL } & \\
\hline $\mathrm{N}$ & mean & std & $\mathrm{N}$ & mean & std & \\
\hline 46 & .2173 & .4170 & 6 & .5 & .5477 & $\begin{array}{c}\mathrm{t}=-1.2186 \\
\mathrm{p}=.2704\end{array}$ \\
\hline \multicolumn{3}{|c|}{ Group A: SGDL } & \multicolumn{3}{|c|}{ Group B: SLDL } & \\
\hline $\mathrm{N}$ & mean & std & $\mathrm{N}$ & mean & std & \\
\hline 6 & .6667 & .5163 & 46 & .4347 & .5012 & $\begin{array}{c}\mathrm{t}=1.038 \\
\mathrm{p}=.3375\end{array}$ \\
\hline \multicolumn{3}{|c|}{ Group A: GDL } & \multicolumn{3}{|c|}{ Group B: MDL } & \\
\hline $\mathrm{N}$ & mean & std & $\mathrm{N}$ & mean & std & \\
\hline 6 & .6667 & .5163 & 14 & .4285 & .5135 & $\begin{array}{c}\mathrm{t}=.9565 \\
\mathrm{p}=.3674\end{array}$ \\
\hline \multicolumn{3}{|c|}{ Group A: MDL } & \multicolumn{3}{|c|}{ Group B: LDL } & \\
\hline $\mathrm{N}$ & mean & std & $\mathrm{N}$ & mean & std & \\
\hline 14 & .3571 & .4972 & 46 & .2173 & .4170 & $\begin{aligned} \mathrm{t} & =.9544 \\
\mathrm{p} & =.3519\end{aligned}$ \\
\hline & Group A & MDL & & roup B: & GDL & \\
\hline $\mathrm{N}$ & mean & std & $\mathrm{N}$ & mean & std & \\
\hline 14 & .3571 & .4972 & 6 & 0 & 0 & $\begin{array}{l}\mathrm{t}=2.6874 \\
\mathrm{p}=.0186\end{array}$ \\
\hline
\end{tabular}

Table 3: Two sample T-test: $M R L$ v.s. $L D L$ (threshold=50\%)

\begin{tabular}{cccccccc}
\hline & \multicolumn{4}{c}{ Group A: MRL } & \multicolumn{5}{c}{ Group B: DL } \\
type & $\mathrm{N}$ & mean & std & $\mathrm{N}$ & mean & std & \\
\hline language & 70 & .3787 & .4888 & 16 & .125 & .3078 & $\begin{array}{c}\mathrm{t}=2.4295 \\
\mathbf{p}=. \mathbf{0 2 1 0}\end{array}$ \\
\hline math & 70 & .1449 & .3546 & 16 & .6667 & .5163 & $\begin{array}{c}\mathrm{t}=-2.0489 \\
\mathrm{p}=.0538\end{array}$ \\
\hline graph & 70 & .4521 & .5011 & 16 & 1 & 0 & $\begin{array}{c}\mathrm{t}=-9.342 \\
\mathbf{p}<. \mathbf{0 0 0 1}\end{array}$ \\
\hline
\end{tabular}

dominated representations has 16 samples. We ran a two-sample T-test between the two groups to compare their performance in answering different type of questions. Results of the comparison are shown in Table 3.

From the eye tracker results, the multiple representation learner group has higher scores in the language questions. But there is no significant difference between the two groups in answering the math questions. A possible explanation of these results is that the language description of the concepts was not straight forward and 


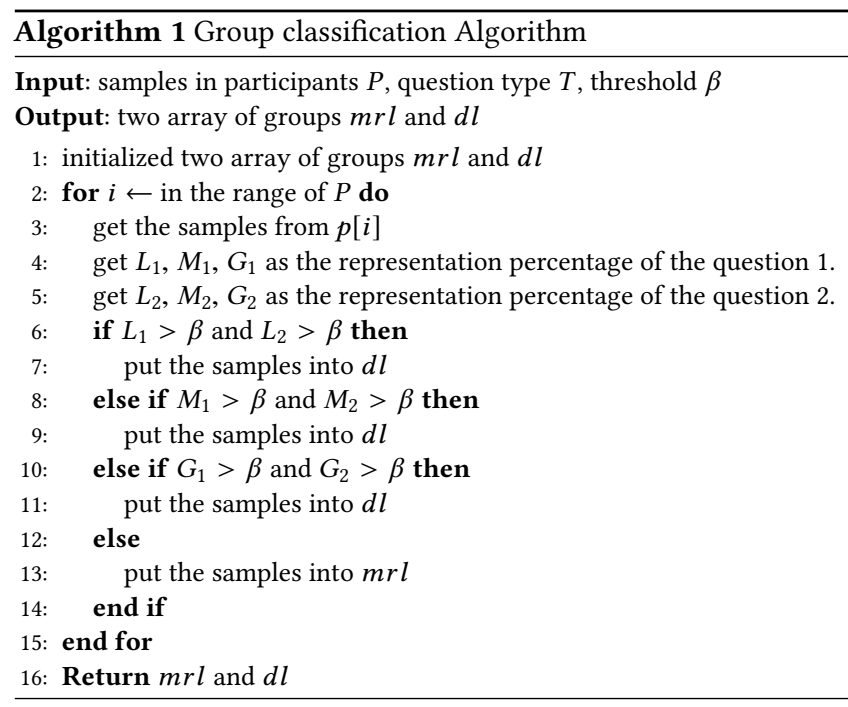

it was hard for the students to create clear mental models. With the aids from other representations, it helped the students to gain better understanding of the concepts in language representation. Graph examples are much more straightforward and the questions are less obscure. So if the learners understand the graph examples, they can find the correct answer fairly easily, without the need to translate the easy to comprehend representation to more abstracted forms. Math notations can be straightforward for the experts but are elusive to the novice learners. Students in the representation dominated groups received higher scores in math questions than the $M R L$ group, This may suggest that if a learner has trouble understanding the abstract math notations, they will resort to other representations to help them understand it better, just like the case of language representations. However, if the translation among math notation and other representations can be much harder. As a result, the assistance from other representations may not be helpful to solve math related questions. This is revealed in the mean quiz scores. When answering language questions, the $M R L$ group had higher average scores than the $D L$ group. But when answering the math and graph questions, the $M R L$ group had lower average scores than the $D L$ group. This may suggest that in the field of cryptography, the math and graph representation can help learners understand text better but text does not seem to help students with math and graph representations.

\subsection{Study Three}

As stated previously in Section 3.2, we are also interested in what sequence the participants use to translate from one representation model to another. From the results of study two, we noticed that multiple representation learners did better in understanding the language representation of the concepts. In study three, we report some observed translating orders that may lead to better performance in understanding language-based concepts.

According to the survey in study one, one of the most common replies in choosing to read the language based representations first is that the knowledge in the real world is mostly carried on language-based media, such as books and papers. And therefore the

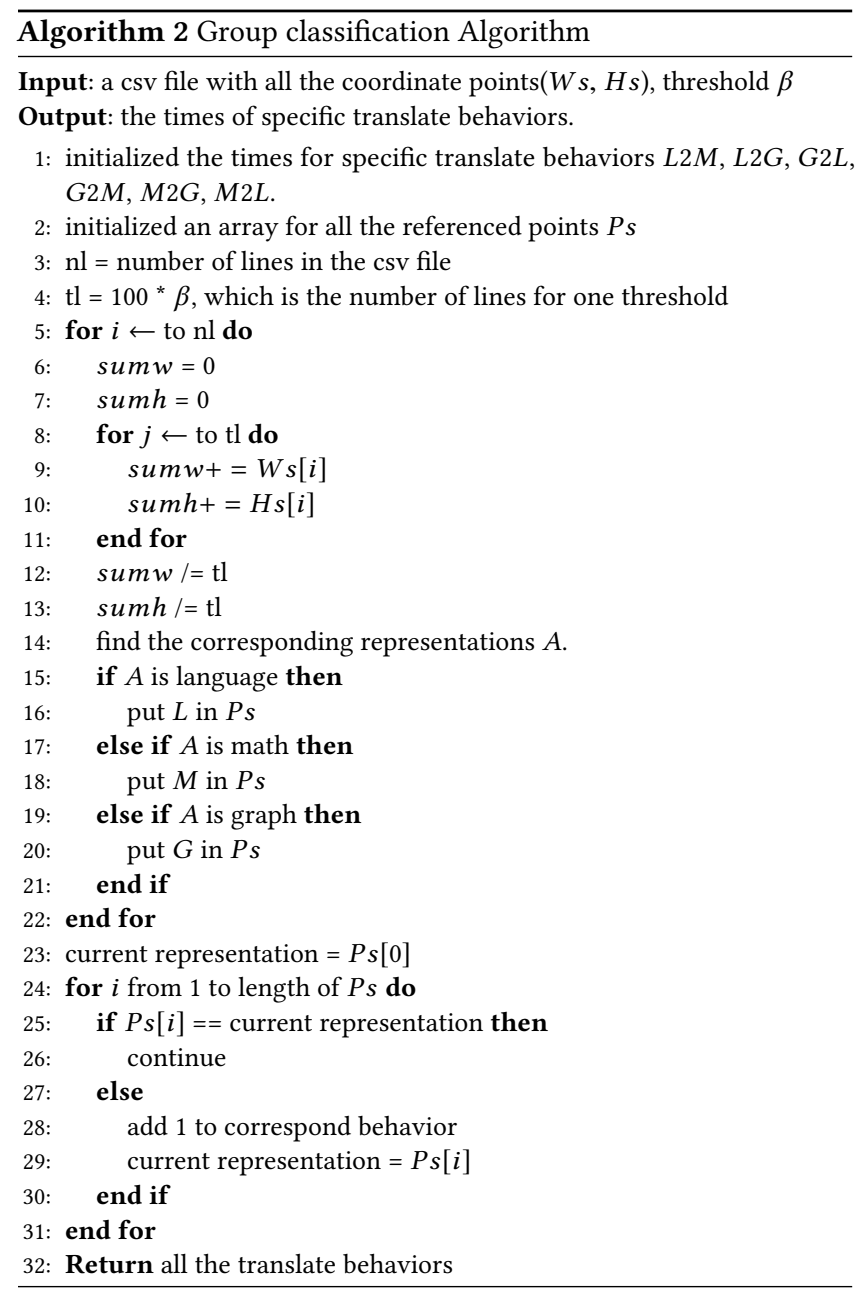

students are used to learning from language-based representations. Results from study two further demonstrated that the languagebased representations on cryptography concepts may not be very straightforward. Learners often found it is necessary to comprehend the concepts from graphs and math notations. In study three, we are trying to explore deeper into the eye tracking recordings to understand if the order of representation transition has any effects on the assessment scores. Specifically, students who read multiple representations in study two outperformed the other group in language the multiple representation groups have better performance in understanding the language representations. Therefore, in study three, we explore if the representation transition orders made any difference in understanding language representation models.

In this experiment, we needed to first observe the translating behaviors. From the perspective of human's reading behaviors, we assumed that the length of read an area, rather than moving the eyes from one area of the screen to another area of the screen, should be longer than several intervals of blinking (0.1s). The eye-tracker recorded 1,000 frames per second. 0.1 s duration contains 100 frames. This is defined as the threshold $\beta$ for the times of the intervals of blinking. We then averaged out the coordinate of these 100 frames as the point of reference for the 0.1 second. If the reference point is 
Table 4: step-wise Regression: scores of language questions v.s. learning behaviors

\begin{tabular}{|c|c|c|c|c|c|}
\hline & attributes & $\mathrm{df}$ & Sum sq & Mean Sq & $\mathrm{F}$ \\
\hline \multirow{12}{*}{ First step } & $\mathrm{L} 2 \mathrm{M}$ & 1 & 1.875 & 1.8746 & \multirow[t]{2}{*}{$8.979(\mathbf{P}=\mathbf{0 . 0 0 3 8 1})$} \\
\hline & Residue & 68 & 14.197 & 0.2088 & \\
\hline & L2G & 1 & 1.787 & 1.7869 & \multirow[t]{2}{*}{$8.506(\mathbf{P}=\mathbf{0 . 0 0 4 7 9})$} \\
\hline & Residue & 68 & 14.285 & 0.2101 & \\
\hline & G2L & 1 & 0.68 & 0.6795 & \multirow[t]{2}{*}{$3.002(\mathrm{P}=0.0877)$} \\
\hline & Residue & 68 & 15.39 & 0.2264 & \\
\hline & G2M & 1 & 0.571 & 0.5714 & \multirow[t]{2}{*}{$2.507(\mathrm{P}=0.118)$} \\
\hline & Residue & 68 & 15.500 & 0.2279 & \\
\hline & M2L & 1 & 0.407 & 0.4066 & \multirow[t]{2}{*}{$1.765(\mathrm{P}=0.188)$} \\
\hline & Residue & 68 & 15.405 & 0.2265 & \\
\hline & M2G & 1 & 0.407 & 0.4066 & \multirow[t]{2}{*}{$1.765(\mathrm{P}=0.188)$} \\
\hline & Residue & 68 & 15.665 & 0.2304 & \\
\hline & attributes & $\mathrm{df}$ & Sum sq & Mean Sq & $\mathrm{F}$ \\
\hline \multirow{12}{*}{ Second step } & L2M & 1 & 1.875 & 1.8746 & \multirow{3}{*}{$\begin{array}{l}9.740(\mathbf{P}=\mathbf{0 . 0 0 2 6 6}) \\
6.766((\mathbf{P}=\mathbf{0 . 0 1 1 4 2})\end{array}$} \\
\hline & $\mathrm{M} 2 \mathrm{G}$ & 1 & 1.302 & 1.3022 & \\
\hline & Residue & 67 & 12.895 & 0.1925 & \\
\hline & L2M & 1 & 1.875 & 1.8746 & $9.740(\mathbf{P}=\mathbf{0 . 0 0 2 6 6})$ \\
\hline & M2L & 1 & 1.302 & 1.3022 & \multirow[t]{2}{*}{$6.766(\mathbf{P}=\mathbf{0 . 0 1 1 4 2})$} \\
\hline & Residue & 67 & 12.895 & 0.1925 & \\
\hline & L2G & 1 & 1.787 & 1.7869 & $9.038(\mathbf{P}=\mathbf{0 . 0 0 3 7 2})$ \\
\hline & G2L & 1 & 1.038 & 1.0375 & \multirow[t]{2}{*}{$5.248(\mathbf{P}=\mathbf{0 . 0 2 5 1 3})$} \\
\hline & Residue & 67 & 13.247 & 0.1977 & \\
\hline & L2G & 1 & 1.787 & 1.7869 & $8.942(\mathbf{P}=\mathbf{0 . 0 0 3 9})$ \\
\hline & G2M & 1 & 0.896 & 0.8960 & $4.484(\mathbf{P}=\mathbf{0 . 0 3 7 9})$ \\
\hline & Residue & 67 & 13.389 & 0.1998 & \\
\hline
\end{tabular}

moved from one representation area to another, we considered a specific translating behavior is observed. In the experiment, we this threshold was set to 1 seond, or 10 blinking times for a participant to read the representation models. The detailed algorithm is illustrated in the Algorithm 2

We then ran a linear regression model on the language questions scores over transition behaviors. In the experiment, we adopted the forward step-wise method [3]. To avoid the accumulation of type 1 error [4], we stopped at the second step. The results are shown in table 4 . From the results, we found that $L 2 M$ and the $L 2 G$ can help to improve the scores for language questions. But on the other way, M2L and G2L didn't contribute much to the test scores on language questions. This might imply that the students raised some questions in reading the language representation models first, and solved the questions after reading the math or graph examples. But they might not realize the question on the other way round. In the second step, we also found that using all three representations could have a significant role in answering the language-based questions. Therefore, the observed ability of representational fluency might be helpful in answering the language questions in our experiment.

\section{CONCLUSIONS}

In this paper, we designed a few eye-tracking experiments to observe the learning behaviors from the students when studying cryptography concepts. From the results, we found that the students who can use more than one representation models can have a better understanding of solving language based questions. But to understand math based or graph based questions, multiple representations did not seem to help as much. A further study also showed that in understanding the language description, the participants can read the language form of the concept first, followed by reading other representation models for a deeper understanding. This means, when teaching cryptography concepts, it may be beneficial to introduce a concept in text first, followed by math notations and graphs.

Future study is much needed to valid the findings from this work with big sample sizes; more assessment questions; and with different age groups. Current study is limited to college students with a good variety of genders and races. In addition, further study is much needed to understand why math and graph representations can help learners on language representations but not the other way around. If we can find a deeper connection among different representations, it may greatly help educators and learners to better learn cryptography concepts as well as other STEM related concepts.

\section{ACKNOWLEDGMENTS}

This project is supported by NSF Award 150046.

\section{REFERENCES}

[1] Martin Abadi and Joan Feigenbaum. 1990. Secure circuit evaluation. Fournal of Cryptology 2, 1 (1990), 1-12

[2] Joseph Beckman, Sumra Bari, Yingije Chen, Melissa Dark, and Baijian Yang. 2017. The Impacts of Representational Fluency on Cognitive Processing of Cryptography Concepts. In The LASER Workshop: Learning from Authoritative Security Experiment Results (LASER 2017). 59-67.

[3] Robert B Bendel and Abdelmonem A Afifi. 1977. Comparison of stopping rules in forward "stepwise" regression. Journal of the American Statistical association 72, 357 (1977), 46-53.

[4] Clemens S Bernhardson. 1975. 375: Type I error rates when multiple comparison procedures follow a significant F test of ANOVA. Biometrics (1975), 229-232.

[5] Hans C Breiter, Scott L Rauch, Kenneth K Kwong, John R Baker, Robert M Weisskoff, David N Kennedy, Adair D Kendrick, Timothy L Davis, Aiping Jiang, Mark S Cohen, et al. 1996. Functional magnetic resonance imaging of symptom provocation in obsessive-compulsive disorder. Archives of general psychiatry 53, 7 (1996), 595-606.

[6] Andrew T Duchowski. 2007. Eye tracking methodology. Theory and practice 328 (2007).

[7] Karen Evans and Franklin Reeder. 2010. A human capital crisis in cybersecurity: Technical proficiency matters. CSIS.

[8] Uriel Feige, Amos Fiat, and Adi Shamir. 1988. Zero-knowledge proofs of identity. Journal of cryptology 1, 2 (1988), 77-94.

[9] Matthew Joel Hill. 2015. Scientific representational fluency: defining, diagnosing, and developing. (2015).

[10] Richard Lesh, Thomas R Post, and Merlyn Behr. 1987. Representations and translations among representations in mathematics learning and problem solving. In Problems of representations in the teaching and learning of mathematics. Lawrence Erlbaum.

[11] Dale C Rowe, Barry M Lunt, and Joseph J Ekstrom. 2011. The role of cyber-security in information technology education. In Proceedings of the 2011 conference on Information technology education. ACM, 113-122.

[12] Xavier Simms and Hongmei Chi. 2011. Enhancing cryptography education via visualization tools. In Proceedings of the 49th Annual Southeast Regional Conference. ACM, 344-345.

[13] Bruce H Thomas and Victor Demczuk. 2000. Evaluation of animation effects to improve indirect manipulation. In User Interface Conference, 2000. AUIC 2000. First Australasian. IEEE, 110-117.

[14] Judith S Zawojewski, Richard A Lesh, and Lyn D English. 2003. A models and modeling perspective on the role of small group learning activities. In Beyond constructivism: Models and modeling perspectives on mathematics problem solving, learning, and teaching. Lawrence Erlbaum Associates, 337-358. 